CASOS CLINICOS

Rev. Chli. Pedlats. 64 (6); 376-380, 1993

\title{
Carcinoma adrenocortical virilizante: tratamiento quirúrgico y quimioterapia
}

\author{
Lautaro Vargas P.1; Ximena Vivanco W. '; Ximena Cuello A. '; Nelly Abodovsky G.1; \\ Francisco Ossandón C.'; Sonia Sirebrenik C. '; Cannen Franco S.
}

\section{Virilizing adrenocortical carcinoma: apparently successful surgical treatment and chemotherapy}

A 3 year old girl was brought to consultation becouse clitcris enlargement. Urine 17 ketosleroid excretion was 102.7 $\mathrm{mg} / 24 \mathrm{~h}$ iNV 1.31 , while urinary dehidroepiandrcslerone (DHA) was $53 \mathrm{mg} / 24 \mathrm{~h}$ (NV 0.5 ) and bolh increased after one month to 285 and $145 \mathrm{mg} / 24 \mathrm{~h}$ respeclively. Selum level of iesloslerone was $177 \mathrm{ng} / \mathrm{dl}(\mathrm{NV} 80$ ) and that of dehidrepiandrosterone sultate (DHEAS) was $59.0 \mu \mathrm{g} / \mathrm{ml}$ i $\mathrm{NV} 0.6 \cdot 3.5)$. A big $(8.7 \times 8.1 \times 10 \mathrm{~cm} /$ adrenal tumor was detected by uliasonograpty and computed axial tomographic scan ICAT\}. The tumor was surgically removed as a whole, in spite of a slighi spilloge al the lower surlace of the liver. Pathology showed fealures of an adrenal carcinoma. Subsecuently she was given Ihree courses of combined lluoruracil, doxorubicin and cisplatinum [FAP] chemotherapy along the next three months. Afler a thee year follow up she has rict clinical neither biochemical evidence of tumor recurrence.

[Key words: adrenal gland, necplasm, carcinomo, virilism.]

Los tumores adrenocorticales son raros. EI primer caso en niños fue informado en 1865. Hasta 1984 se habían comunicado 300 , considerando adenomas y carcinomas ${ }^{1}$. El carcinoma adrenocortical representa $6 \%$ de los tumores adrenales pediátricos ${ }^{2}$. El grupo cooperativo oncológico británico (UKCCSG) había registrado, hasta 1986,8173 casos de cánceres en menores de 15 años, sólo $17(0,2 \%)$ eran de carcinoma suprarrenal, lo que coincide con otras series en que la incidencia es de $0,19 \%$ de todos los tumores malignos en menores de 15 años $3-5$.

Aproximadamente en $58 \%$ de los pacientes pediátricos con tumores supratenales éstos son funcionantes ${ }^{2}$. De 222 casos publicados en ninos, un tercio tenia virilización como signo preponderante y otro tercio hipercortisolismo. También ha sido descrito una variedad de otros sín-

1. Universidad de Chile, División Ciencias Médicas Occidente. Departamento de Pediatría y Cirugía Infantil, Servicio de Cirugía Infantil y Pediatria, Servicio de Anatomia Palológica, Unidad de Endocrinología y Hemalología - Oncologia, Hospital San Juan de Dios. tomas complejos incluyendo feminización e hiperaldosteronismo ${ }^{1}$. La mayoría de los pacientes son mujeres, en proporción mujer : hombres de 2, 2:16. El promedio de edad al diagnóstico es 4,63 años (márgenes de 5 días a 16,5 años) 7 .

Existe mucha controversia sobre el diagnostico diferencial entre adenoma y carcinoma. Los criterios más utilizados son el tamaño y el peso del tumor, las características histológicas de malignidad y las concentraciones hormonales ${ }^{1,3}$. El conjunto de estos hallazgos debe considerarse para fundanentar el diagnóstico de matignidad. Otro motivo de discusión es la utilidad de la quimioterapia adyuvante ${ }^{\beta}$ que tradicionalmente desde 1960 se realiza con mitotano (o.p" DDD) 9 .

El caso siguiente es el đe una niña de tres años afectada por un carcinoma suprarenal y signos de virilización, que pudo ser extirpado totalmente, en la cual se empleó quimiotcrapia adyuvante con fluorouracilo, doxorrubicina y cisplatino (FAP) ${ }^{10}$. No hemos encontrado comunicaciones previas con este tratamiento en la literatura pediátrica chilena y sólo conocemos un caso en un menor de 15 antos, que no ha sido publicado. 


\section{Caso clínico}

Niña de tres añus dos meses, consultó en julio de 1989, por aumento progresivo del clítoris en 2 meses de evolución. Talla y peso en percentil 75, aumento del desarcollo mus. culat, piel con pigmentación nomal. presión arterial nomal, mejillas rubicundas, bozo incipiente. No se palpaban masas abdominales. Vello pubiane tipo lanugo, clítoris de $1,8 \mathrm{~cm}$ de largo por $1 \mathrm{~cm}$ de ancho. Edad ósea 3 años 4 meses. In orina de $24 \mathrm{~h}: 17$ keloesteroides (17 ks) $102,7 \mathrm{mg} / 24 \mathrm{~h}$ (VN 1 a 3) y dehidroepiandrosteron (DHA) $53 \mathrm{mg} / 24 \mathrm{~h}$ (VN 0,5 ), los que después de un mes aumentaron a 285 y 145 $\mathrm{mg} / 24$ h respectivamente. Contisol libre $y 17$ hidroxiesteroides urinarios nomales. En el suero, lestostcrona $177 \mathrm{ng} / \mathrm{dl}$ (VX 80) y dehidroepiandtosterona sulf ato (DHEAS) 59,6 tg $/ \mathrm{d}$ ( (VN 0,6 - 3.5). Estradiol plasmático y ritmo circadiano the cortisol nomales. La ecotomografía abdominal mostró una gran masa suprarrenal derecha de ecogenicidad irregular (múltiples áreas ecogénicas pequeñas en su interior). La vena cava estaba comprimida y desplazada hacia adelante pero era permeable en las mediciones Dappler La tomografía axial computadorizada (TAC) confimó que había una masa saprarrensl derecha (de $8.7 \times 8.1 \times 10 \mathrm{~cm}$ ) de densidad heterogénea, con zonas de menor densidad en su interior (figura 1), que estaba en íntima contacto con el hígado y desplazaba el rin̄ón en dirección caudal, la vena cava inférior hacia adelante y el páncreas hacia la izquierda. La cintigrafía con DMSA mostró desplazamiento caudal de! riñón derecho y leve deformidad de su polo superior. Fue sometida a toracolaparotomia, encontrándose un gran tumor suprarrenal de $12 \mathrm{~cm}$ de diámeiro que se liber $\delta$ laboriosamente de la cara inferior del hígado, a la que se adheria intimamente, con suspecha de infultración de la cápsala hepática. Al realizar la disección, se produjo una rơura discreta, pero sl tumor fue extifpado tolalmente (figura 2). En la inlervención nu se encontrajon signos de metástasis abdominales, hepáticas o en los ganglios retroperito. neales.

El tumor era de superficie pardo rojiza, bien delimitado, exceplo en el área correspondiente al higado, que se cncontraba macerada. Pesó l $080 \mathrm{~g}$. En el conce era amarillento con áreas rojizas y friable. Estaba constituido mayormente por células con abundante citoplasma eosinótilo y núcleo redondeado central de cstructura homogénca, dispucstas en cordones o trabéculas. En algunas áreas se observaba extensa anaplasia, núcleos irregulares hipercromáticos, algunos de forma cerebroídea. Había algunas células aisladas con necrosis. No se comprobó penetración vascular o infiltraciŏn je la cápsula por el tumor (figura 3). Se envió una muestra a) servicio de anatomía patológica del "St. Jude ChiJdren's Research Hospical" (Dr. Jesse Yenkis y Dr. Gastón Berard), donde se corroboró el Jiagnóstico de carcinoma cortico. adrenal).

Puesto que durante la intervención se habla producido ruplura de la cápsula al desprender la masa de la cara inferior del hígado, se decidió realizar quimioterapia adyuvante. Por no disponer en el país de milotanot. 11 , se utilizó el es. quema FAPio, en Ires curas, pues se consideró que el tumor estaba en etapa I. La quimioterapia fue aceptablemente tolerada, observándose sólo vórnitos, cquivalente a escala 3 de roxjcidad (National Cancer Institute). En el postoperato rio se observ6 dramática nomalización de los esteroides suprarrenales plasmáticos y urinarios. l.os $17 \mathbf{k}$ s urizarios y

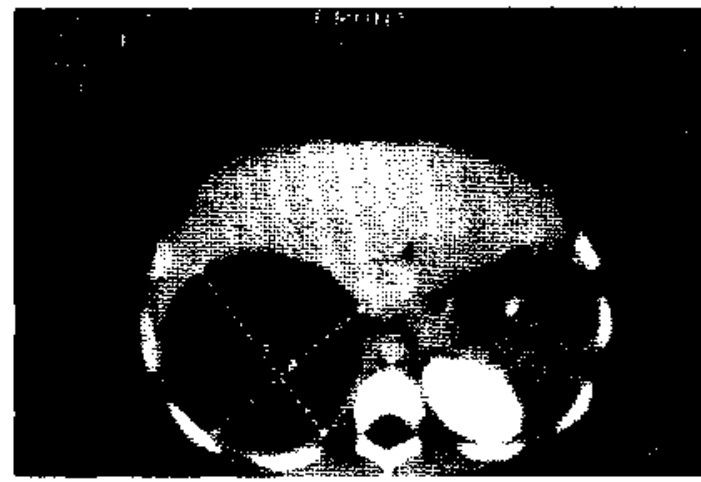

Fig. 1: Tomografia axial computadorizada del abdomen Se registra uлa gran masa suprarrenal derecha dc densidid ultrasonogrăfica heterogénea.

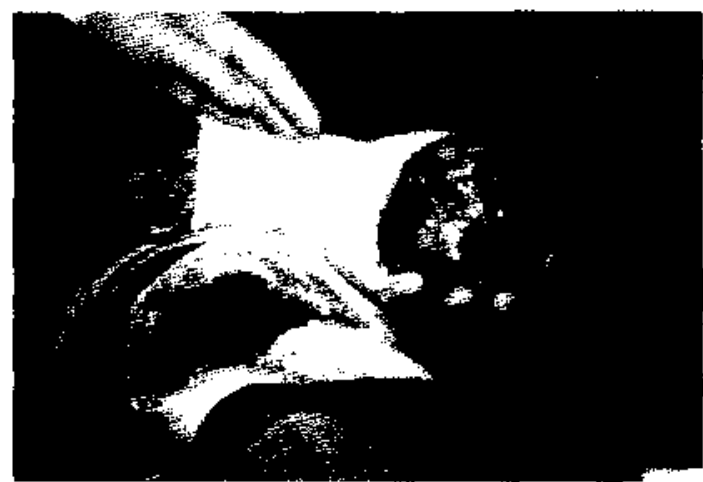

Fig. 2: Aspecto macroscópico del tumor supranenal de 12 cm de djámetro, una vez extraído.

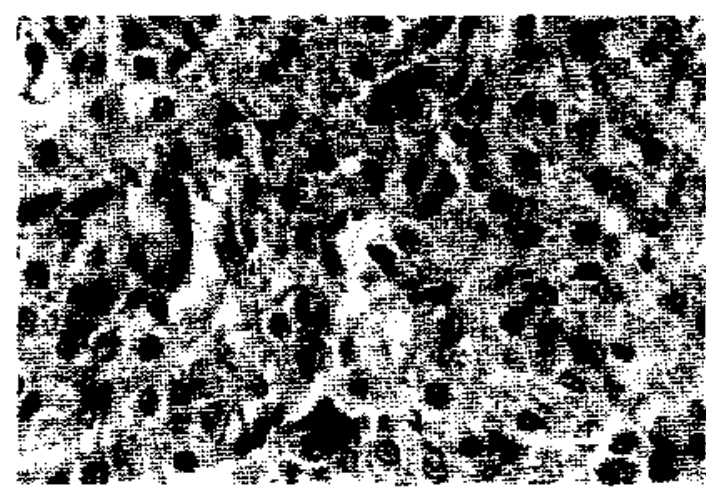

Fig. 3: Histopatología del tumor extirpado. Tinción hematoxilina eosina, $400 \mathrm{X}$. Células con abundante citoplasma eosinófito, núcleo redondeado central de estructura homogénea, dispuestas en cordones o trabéculas. En algunas ăreas hay cxtensas a naplasia, núcleos irreguares $\mathrm{e}$ hipercromáticos y necrosis celular sislada 
andrógenos plasmáticos se han mantenido nomales hasta el momento del envio de esta comunicación; la concentración de ACTH plasmático, por RLA, era $54 \mathrm{pg} / \mathrm{ml}$ (normal); el desarrollo físico y psicomolor ha sido nomal: so estatura se ha mantenido en el percentil 75 y el tamaño del clítoris se ha reducido parcialmente.

\section{Comentario}

La mayoría de los tumores suprarrenales ocuren en niños menores de cinco años y también existen casos congénilos ${ }^{2}$. Se ha descrito su asociación con diversas anomalías congenitas, como hemihipentrofia, lesiones cutáneas, síndrome de Beckwith Wiedemann 1,12 .

Los signos de virilización plantean el diagnóstico diferencial con hiperplasia suprarenal congénita (HSRC). En muchos casos se combinan los signos de virilización con los de hipercortisolismo, constituyendo un sindrome de Cushing 3, 9, 13. Otros síndromes endocrinos menos frecuentes son feminización por hipersecreción de estrógenos ${ }^{2}$, ginecomastía ${ }^{15}$, hiperaldosteronismo 14, 16, 17. alcalosis hipokalémica por exceso de deoxicorticosterona y síndromes complejos de feminización y masculinización simultánea ${ }^{7,13,18 .}$

En ninas con virilización se deben medir las hormonas séricas y urinarias y hacer prueba de estimulación con ACTH para diferenciarlas de casos con hiperplasia suprarrenal congénita. Los estudios de imágenes son fundamentales, dado que sólo $50 \%$ de los tumores suprarrenales se palpan en el momento del diagnóstico, debido a su situación anatómica. La ecotomografía y la tomografía axial son los procedimientos más útiles para precisar el tamaño, fa extensión del tumor, su relación con estructuras vecinas y así encontrar signos de malignidad y programar la cirugía. Uno de los criterios más utilizados para diferenciar entre adenoma benigno y carcinoma es el tamaño y peso del tumor, pues la mayoria de los adenomas pesan menos de $100 \mathrm{~g}$ (promedio $20 \mathrm{~g}$, márgenes 6 a $126 \mathrm{~g}$ ), mientras los carcinomas pesan, en promedio, $508 \mathrm{~g}$ y varían de 30 a $4500 \mathrm{~g}$. Otros indicadores de malignidad son los histológicos: actividad mitótica aumentada, pleomorfismo celular y nuclear, calcificación, hemorragias, necrosis e invasión de la cápsula y de los vasos sanguíneos. Todos estos hallazgos son más frecuentes en los carcinomas, pero pueden verse en los tumores benignos de los niños, con mayor frecuencia que en los adultos 19.20 . En nuestro paciente, el tamaño del tumor, su peso, la adherencia a la cápsula hepática, la presencia de atipias y necrosis celulares al examen histológico fueron criterios indicativos de malignidad.

La magnitud de la excreción de $17 \mathrm{ks} \mathrm{cs}$, para algunos autores, indicador de carcinoma cuando es muy alta ${ }^{21}$. En los adenomas los $17 \mathrm{ks}$ en la orina no superan $70 \mathrm{mg}$ en $24 \mathrm{~h}$, mientras en los carcinomas pueden subir hasta $800 \mathrm{mg} / 24 \mathrm{~h}$, si bien algunos casos evolucionan con cifras similares a las registradas en adenomas, por lo que este signo sólo sería útil cuando los valores están muy elevados. La determinación de] DNA nuclear mediante citometria de flujo podría ser de utilidad como indicador de recurrencias y malignidad, especialınente en casos difíciles y ambiguos, al demostrar aneuploidía ${ }^{22}$.

El pronóstico de los pacientes con estos tumores es malo, especialmente en lesiones muy grandes con invasión local, metástasis o irresecables. Las recaídas son muy precoces y la afección es habitualmente letal: de 127 niños afectados con seguimiento registrado, sólo $23 \mathrm{han}$ sobrevivido más de dos años, aunque sólo 34 muertes han sido ocasionadas por recurrencias del tumor. Muchos fallecimientos mencionados en la literatura antigua fueron consecuencia de complicaciones postoperatorias o inadecuado reemplazo esteroidal. En las series más recientes los resultados parecen más promisorios, con escasas recaidas al cabo de 1 ó 2 años después de la extirpación quirúrgical.

No hay acuerdo respecto a la efeclividad de la quimioterapia adyuvante. El mitotano induce regresión del tumor en 34 a $61 \%$ de los pacientes y mejoría en 50 a $85 \%, 10,23,24$. Sin embargo, la mayoría de las respuestas son parciales y se mantienen, en promedios, sólo por 10 a 12 meses $^{10}$. Por otra parte, este medicamento produce efectos adversos importantes, entre ellos insuficiencia suprarrenal y gonadal, que obligan a hacer tratamicntos de reemplazo, lo que resta efectividad a la vigilancia de la tasa de $17 \mathrm{ks}$ urinarios en la detección de las recaídas ${ }^{19}$. La búsqueda de otros regímenes quimioterápicos no ha dado, hasta ahora, resultados satisfactorios. Con la combinación de fluorouracilo, doxorrubicina y cisplatino (FAP) se ha logrado remisión completa, mantenida durante 38 meses, en un paciente con carcinoma suprarrenal y metástasis pulmonares ${ }^{10}$. Basados en csa experiencia diseñamos tres curas 
con FAP para nuestra enferma, ya que durante el proccdimicnto quirúrgico se sospechó que había infiltración hepática y debido a la ruptura de la cápsula del tumor al desprendcrlo de la cara inferior del hígado. La tolerancia de este esquema fue aceplable; no se han detectado secuelas de ningún tipo $\mathrm{y}$ la paciente ha cvolucionado en las condiciones satisfactorias descritas en la prescntación de su caso hasta transcurridos tres años desde la quimioterapia.

\section{Resumen}

Una niña de tres años consultó por crecimicnto del clítoris. La excresión urinaria de 17 ketoesteroides (17 ks) era $102,7 \mathrm{mg} / 24 \mathrm{~h}$ (VN 1 - 3) y la de dehidroepiandrosterona (DHA) $53 \mathrm{mg} / 24 \mathrm{~h}$ (VN 0,5) al ingreso; ambas aumentaron un mes después a 285 y $145 \mathrm{mg} / 24 \mathrm{~h}$ respectivamente. Cortisol libre y 17 hidroxiesteroides urinarios normales. En el sucro, testosterona $177 \mathrm{ng} / \mathrm{dl}$ (VN 80) y dehidroepiandrosterona sulfato (DHAS) $59,6 \mu \mathrm{g} / \mathrm{ml}$ (VN 0,6 - 3,5). La ecotomografía abdominal y la tomografía axial computadorizada mostraron signos de un tumor suprarrenal derecho de $8,7 \times 8,1 \times 10 \mathrm{~cm}$, que tue extirpado quirúrgicamente en su totalidad, produciéndose una discreta ruptura al desprenderlo de la cara inferior del hígado. En el postoperalorio se normalizaron los andrógenos suprarrenales séricos y urinarios. Los exámenes histopatológicos confirmaron que se tralaba de un carcinoma suprarrenal; consecuentemente recibió ures ciclos de quimioterapia con fluorouracilo, doxorrubicina y cisplatino (FAP) a lo largo de tres meses. Tres años después el desarrollo ponderal y estatural, las concentraciones séricas de $\mathrm{ACTH}$, de andrógenos en suero y orina y los exámenes ecotomográficos correspondientes cran normales.

(Palabras clave: glándulas suprarrenales, neoplasia, carcinoma, viriljzación.)

\section{Referencias}

I. Lee $P$ h, Winter $R$, Green $O$ : Virilizing adrenocontical utrors in childhood: cighl cases and a review of the literalure. Pediatrics 1985: 76: 437-444.

2. Saracco $S$, Abramowshy $C$, Taylor $S$ et at : Sponta. meously regressing adrenocontical carcinoma in a ncwborn. Cáncer 1988: 62: 507-511.
3. Prast Ch, Douglass E: Managemenl of the tess common cancers of childhood in Principics and practice of Pe. diauric uncology editado por Pizzo PL., Poplack. J.B. Lippincott. Philadelphia 1988; 759-782.

4. L'nited Kingdom. Children's Cáncer Study Group UKCCSG. Scientific Repor1 1988.

5. Young J, Ries L, Silverberg E, et at.: Cáncer, incidence. survival, and monality for children younger than age 15 years. Cáncer 1986; 58: 598-602.

6. Hayles A, Hahn H, Spragne RG, ef al.: Hormonesccreting tumors of the adrenal cortex in children. Pediatrics 1966; 37: 19-25.

7. Neblet $W$, Frexes-Steed $M$, Scout $W$ : Experience with adrenocortical neoplasms in childhood. The American Surgeon 1987: 53: 117-125.

8. Luton J, Cerdes S, Billand $L$, et al.: Clinical features of adrenocortical carcinoma, prognustic factors, and the effect of milotane therapy. N Engl J Med 1990; 322: 1201.

9. Vuisman $S$, Pizzi T, Rivera $G$, et al, . Adrenocarcinoma suprarrenal. Rev Chíl Pediar 1971; 42: 175-181.

10. Schlumberger $M$, Ostronoff $M$, Bellaide $M$, et al.: 5 fuoracil, doxonibicin and cisplatin régimen in adrenal cortical carcinoma. Cáncer 1988: 61: 1492-1494.

11. Erikson B, $O^{\prime}$ berg $K$, Cursteds $T$, et al: Treatument of hornone-producing adrenocortical cáncer with o,p'DDD and streptozocin. Cáncer 1987; 59: 1398. 1403.

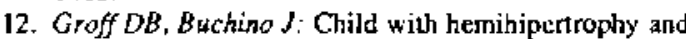
a right flank mass. J Pediatr 1982; I00: 500-504.

13. Gitbert $M G$. Cleveland WW: Cushing's syndrome in unfancy. Pediatrics 1970; 46: 217-229.

14. Arteaga E, Biglieri $E G$, Kater $C E$ : Aldosterone-producing adrenocortical carcinoma: preoperative recugnition and course in three casses. Ann Intern Med 1984: 101: 316-321.

15. Itami RM. Admundson GM. Kaplan SA, et al.. Prepuberal Gynecumastia caused hy an adrenal cumor. An 1 Dis Child 1982; 136: 584-586.

16. Ganguly A. Bergstein J, Grim C: Childhood primary aldosteronism due to an adrenal adenoma: preoperative Iocalization by adrenal vein catheterization. Pediatries 1980; 65: 605 .

17. Powell Jackson J, Calin A, Fraser $R$, et al.: Excess deoxycorticosteronc secreting from adrenocortical carcinoma. Br Med J 1974; 2: 32.

18. Bacon GE. Lowrey $G$ : Ferrinizing adrenat tumor in a six year old boy. J Clin Endocrinol Metab 1965; 25 . $1403-1406$

19. Freeman D: Steroid humone-producing tumors in man. Endocrine Review 1986; 7: 204-220.

20. Cagh Ph, Ilougih A, Pysher J, et al: : Comparison of adrenal cortical tumors in children and adults. Câncer $1986 ; 57: 2235-2237$.

21. Michaud P. Andrade J: Carcinoma Suprarrenal virilizante de niño. Rev Chil Pediatr 1969; 40: 790793.

22. Amberson $J$, Vatighan $D$, Gray $G$, Naus $G$. Flow cytometric analysis of nuclear DNA form adrenocortical neoplasms. Cáncer 1987; 59: 2091-2095.

23. Lubilz $J$, Freemon $L_{1}$ Okwn $R$ : Mitolane in inoperable adrenal cortical carcinoma. JAMA 1973; 223: 1109 IIII. 
24. Eriksson B, Oberg K, Curstedt Th: Trealment of hormoneproducing adrenocortical cáncer with o,p'DDD and streptozocin. Cáncer 1987; 59; 1398 1403.
25. Bertagna Chr, Orth D: Clinical and laboratory findings and resules of hieraphy in 58 patients with adrenocortical tumors admitted to a single Medical Center (1951 10 1978). Am J Med 1981: $71: 855.875$. 